\title{
Atypical Spleen Hemophagocytic Histiocytic Sarcoma in a Dog
}

\author{
Heloize Stein', Mariana Pires de Oliveira', Camila Serina Lasta', Juliana Eckert' ${ }^{2}$, Juliana Sesana Coradini ${ }^{3}$, \\ Letícia Engelhardt Luz ${ }^{3}$, Emerson Antônio Contesini ${ }_{(\mathbb{D})}^{4}$ \& Clairton Marcolongo-Pereira ${ }_{\mathbb{C}}{ }^{3}$
}

\begin{abstract}
Background: The histiocytic sarcoma (HS) complex is a set of malignant neoplasms originating from interstitial dendritic cells or macrophages. When it involves macrophages of the splenic red pulp and bone marrow, it is referred to as hemophagocytic histiocytic sarcoma (HHS). HHS behaves more aggressively than HS and is usually fatal. HHS can be diagnosed by cytological and histopathological examination of neoplastic tissue. HHS is confirmed by immunohistochemistry using an anti-CD11d antibody. This neoplasm is often confused with immune-mediated hemolytic anemia or Evans syndrome due to erythrophagocytosis and platelet consumption. The clinical presentation of the animals progresses with evident anemia and thrombocytopenia, leading to signs such as prostration, inappetence, and pale mucosa, making diagnosis challenging and often late. This study aimed to report the clinic-pathological aspects of a canine with atypical hemophagocytic splenic HS.

Case: A 4-year-old male Shih-Tzu canine was referred to the Veterinary Hospital with a history of prostration and anorexia. Pale mucous membranes were observed on physical examination. Blood tests revealed non-regenerative anemia, leukopenia, and thrombocytopenia. Serum protein levels were below the reference values for the species in biochemical examinations. Hemoparasitosis was suspected; however, the result of the polymerase chain reaction was negative. Abdominal ultrasound revealed a splenomegaly with heterogeneous parenchyma and a slightly irregular surface, but no visible mass in the spleen. Due to the difficulty of stabilizing the patient, even after successive transfusions, the animal underwent exploratory laparotomy with medial access and posterior splenectomy. Subsequently, the spleen was surgically removed, fixed in $10 \%$ buffered formalin, and processed routinely. Macroscopically, it had an irregular reddish-brown capsular surface. Histopathological examination of the spleen revealed a densely cellular neoplasm composed of round to spindle cells (histiocytes) arranged haphazardly in variably sized sheets separating the pre-existing spleen stroma. These histopathological findings were consistent with a histiocytic malignant neoplasm. Immunohistochemical analysis was performed to better define the origin of the histiocytic neoplasm. Neoplastic cells showed positive immunostaining of more than $80 \%$ of tumor cells for the CD11d antibody and weak immunostaining for CD11c and lysozyme. The patient survived for less than 30 days after the first hospital visit.

Discussion: The diagnosis of HHS was based on the histological characteristics and positive immunostaining of more than $80 \%$ of the tumor cells for the CD11d antibody. HHS is an extremely aggressive and rare tumor that affects elderly dogs of any breed. In this study, HHS had atypical histologic characteristics, in which erythrophagocytosis and hemosiderin were not observed within macrophages. HHSs arise from macrophages of the red pulp of the spleen or bone marrow and express the $\mathrm{b} 2$ integrin, $\mathrm{CD} 11 \mathrm{~d}$, and have low expression of $\mathrm{CD} 1$ and $\mathrm{CD} 11 \mathrm{c}$, which are predominantly expressed by nonhemophagocytic HS. The hematological and biochemical changes observed in this case were similar to those described in other dogs with HHS. Treatment of HHS is only palliative. Erlichia ewingii, E. canis, Anaplasma phagocytophilum, A. platys, Borrelia burgdorferi, Dirofilaria immitis, Leishmania infantum and immune-mediated hemolytic anemia are the main differential diagnoses because they cause anemia and thrombocytopenia accompanied by splenomegaly.
\end{abstract}

Keywords: histiocytic sarcoma, spleen, immunohistochemistry, splenectomy, erythrophagocytosis. 


\section{INTRODUCTION}

Histiocytic tumors in dogs represent a group of neoplasms with similar morphological characteristics but with different cellular origins and biological behaviors $[3,13]$. Among these tumors, histiocytic sarcomas (HS) are a complex of malignant neoplasms that, when they originate from interstitial dendritic cells, can be classified as localized or disseminated, depending on the involvement of multiple sites; if they have a macrophagic origin, they are called hemophagocytic histiocytic sarcomas (HHS) [2,8].

As other complex neoplasms, HHS affects middle-aged to elderly animals, with no predilection for sex $[1,8]$, and it can affect dogs of any breed, but the most predisposed are the Bernese Mountain Dog, Rottweiler, flat-coated Retriever [5,8,12], and Miniature Schnauzer [11].

HHS involves the splenic red pulp and bone marrow, usually without the formation of massive lesions at these sites and can later extend to the liver and lungs via the intravascular route [9]. A frequent finding is diffuse splenomegaly [8], which is associated with non-specific signs, including anorexia, weight loss, lethargy, and pale mucous membranes $[1,8]$.

A presumptive diagnosis can be obtained by cytological and histopathological examinations and must be confirmed by immunohistochemistry $[10,11]$. No effective treatment for HHS has been established [3]. Currently, the same drugs adopted for the variant of dendritic cells are used, but HHS is associated with the worst prognosis of all histiocytic disorders, with a survival time of 1 to 2 months [6].

This study aimed to report the clinic-pathological aspects of a canine with atypical hemophagocytic splenic HS.

\section{CASE}

A 4-year-old male Shih-Tzu, weighing $6.8 \mathrm{~kg}$, was referred to a private Veterinary Hospital with a history of prostration and anorexia. The patient had an up-to-date vaccination history and control of endoparasites and ectoparasites. In the anamnesis, the tutors reported that the canine had recently visited a dog hotel and had since presented these changes.

Physical examination revealed pale mucous membranes; systolic blood pressure $200 \mathrm{~mm} / \mathrm{Hg}$, measured by the Doppler method; and $6.0 \mathrm{mmol} / \mathrm{L}$ lactate (reference value $[\mathrm{RV}]:<3.2$. The other parameters were within the normal range for the species. The patient was hospitalized for complete blood count, creatinine, potassium, abdominal ultrasound, and investigation of blood parasites by polymerase chain reaction (PCR).

The hemogram showed normocytic, normochromic anemia, non-regenerative anemia, mild anisocytosis, and polychromasia. In addition, the animal had hypoproteinemia (4.6 g/dL; RV: 6.0-8.0 g/dL), severe leukopenia (1,700 total leukocytes; RV: 6,000$17,000 / \mathrm{uL})$, thrombocytopenia $(<20,000 / \mathrm{uL})$ platelets; RV: 200,000-500,000/uL) and hypokalemia (3.3 $\mathrm{mmol} / \mathrm{L}$; RV: $3.5-5.1 \mathrm{mmol} / \mathrm{L})$. PCR was performed for Anaplasma spp., Babesia spp., Leishmania spp., and Rangelia vitalii, and the results were negative.

Abdominal ultrasound showed an enlarged spleen with convex margins, defined contours, slightly irregular surface, normoechoic parenchyma, and slightly dilated lienal veins, suggesting a neoplastic process (Figure 1A and 1B). Due to the difficulty of stabilizing the patient, even after successive transfusions, the animal underwent exploratory laparotomy with medial access and posterior splenectomy.

Subsequently, the spleen was surgically removed, fixed in $10 \%$ buffered formalin, processed routinely, embedded in liquid paraffin, cut at $5 \mu \mathrm{m}$, and stained with Hematoxylin and Eosin ${ }^{1}$. Macroscopically, it had a reddish-brown capsular surface, was smooth, irregular, and averaged $21.0 \times 7.3 \times 1.5 \mathrm{~cm}$. Microscopically, replacing the splenic parenchyma architecture was a poorly demarcated, infiltrative, densely cellular neoplasm composed of round to spindle cells (histiocytes) arranged haphazardly in variably sized sheets separating the pre-existing spleen stroma. Neoplastic cells have variably distinct cell borders: moderate to abundant eosinophilic, granular, often microvacuolated with foamy cytoplasm, and irregularly round to reniform nuclei with hyperchromatic chromatin. Anisocytosis and anisokaryosis were observed. The mitotic rate is variable with up to 2 per high-power field $\left(2.37 \mathrm{~mm}^{2}\right)$ [Figure 2].

Tissue samples were subjected to immunohistochemistry using markers for $\mathrm{CD}^{2}, \mathrm{CD}^{2}, \mathrm{CD} 10^{2}$, CD11 ${ }^{2}, \mathrm{CD}_{11}{ }^{2}, \mathrm{CD} 31^{2}, \mathrm{CD}^{2} \mathrm{a}^{2}, \mathrm{MUM}^{2}, \mathrm{PAX}^{2}$, granzyme ${ }^{2}$, and lysozyme ${ }^{2}$. This technique was performed in a private laboratory. The tested samples were negative for CD3, CD79a, MUM1, PAX5, CD5, CD10, CD 31, and granzyme but showed strong immunostaining for CD11d (Figure 3A) and weak immunostaining 


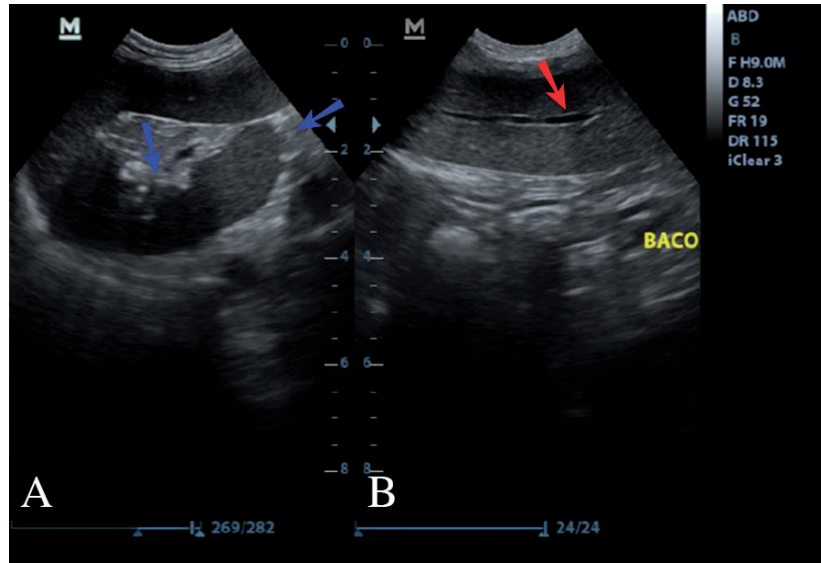

Figure 1. Ultrasonographic image of the spleen in a dog with hemophagocytic histiocytic sarcoma. A- Spleen with a slightly irregular surface and bulging margins (blue arrows). B- Dilated lienal veins (red arrow).

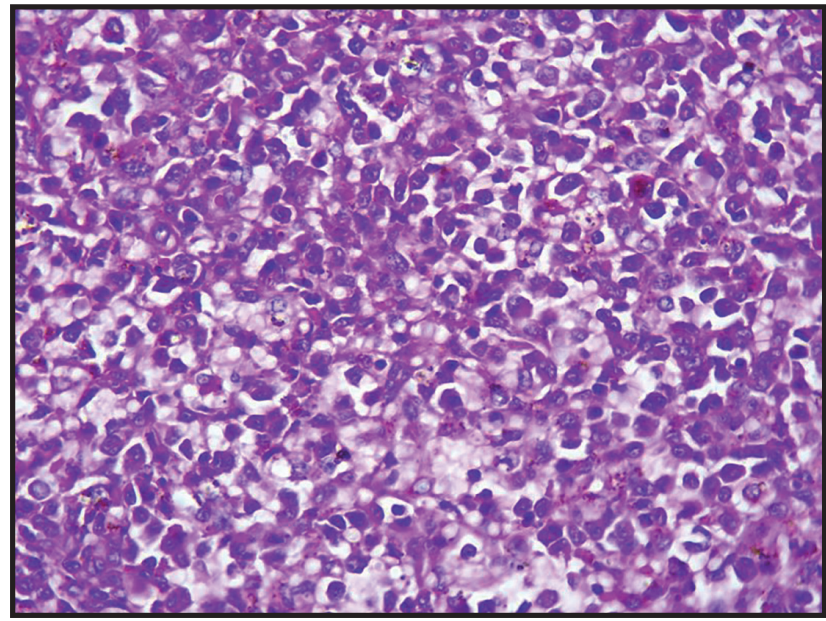

Figure 2. Histopathology of the spleen with hemophagocytic histiocytic sarcoma. Infiltrative neoplastic histiocytes are obliterating spleen architecture $[\mathrm{H} \& \mathrm{E} ; 40 \mathrm{x}]$

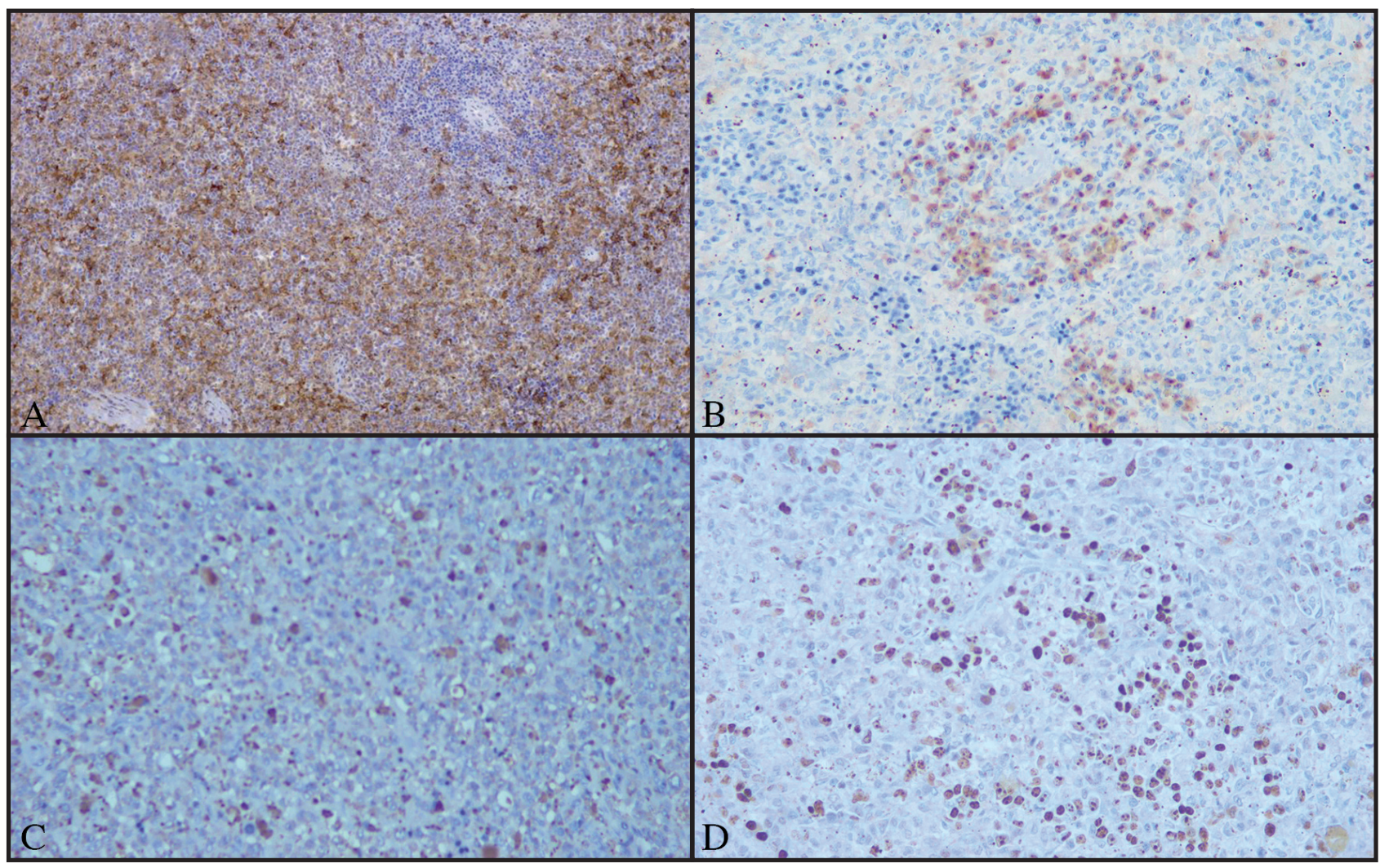

Figure 3. Immunohistochemical analysis of the spleen with hemophagocytic histiocytic sarcoma (HHS). A- Neoplastic histiocytes show strong immunostaining for CD11d. B- Weak immunostaining for CD11c. C- Weak immunostaining for lysozyme. D- Approximately $30 \%$ of neoplastic cells immunostained for Ki67.

for CD11c (Figure 3B) and lysozyme (Figure 3C). In addition, approximately $30 \%$ of the neoplastic cells were positive for Ki67 (Figure 3D).

The patient was discharged after 7 days. However, 26 days after the first visit and 20 days after splenectomy, he returned with complications in the clinical picture, with $5.8 \mathrm{mmol} / \mathrm{L}$ of lactate, systolic blood pressure $210 \mathrm{~mm} / \mathrm{Hg}$, pale mucous membrane, temperature of $39.7^{\circ} \mathrm{C}$, tachycardia and tachypnea, and died without being able to start a chemotherapy protocol. The owner did not allow necropsy to be performed.

\section{DISCUSSION}

The diagnosis of HHS was based on positive immunostaining of more than $80 \%$ of the tumor cells for the CD11d antibody, little staining for CD11c, and 
hematological alterations. HHSs arise from macrophages of the red pulp of the spleen or bone marrow and express b2 integrin, CD11d, and have low expression of CD1 and CD11c, which are predominantly expressed by non-hemophagocytic HS in canines [9]. Furthermore, HHS must be differentiated from hepatosplenic $\mathrm{T}$ cell lymphoma, which may have similar clinical features and express b2 integrin [7]. In this case, the tumor was negative for several immunohistochemical markers of lymphoma.

The hematological changes observed in this case were similar to those described in other dogs with HHS $[1,9,12]$. Anemia and leukopenia observed in patients with this type of tumor are associated with the activity of mutated macrophages, which perform phagocytosis in lymphoid organs, and the absence of regeneration can be explained by the infiltration of neoplastic histiocytes into the bone marrow [8,9]; however, it was not possible to perform the myelogram to confirm this hypothesis. Thrombocytopenia is probably derived from multiple causes, including consumption and phagocytosis by neoplastic macrophages. Other causes of anemia and thrombocytopenia accompanied by splenomegaly, such as hemoparasitosis and immune-mediated hemolytic diseases, were ruled out. Erlichia ewingii, E. canis, Anaplasma phagocytophilum, A. platys, Borrelia burgdorferi, Dirofilaria immitis, Leishmania infantum, and IMHA are the main differential diagnoses [8-10].

Marked hypoproteinemia and hypokalemia were observed in this case. Biochemical alterations can be observed in dogs with HHS, mainly hypoalbuminemia, hypercholesterolemia, and hypoproteinemia, secondary to the inflammatory process induced by the neoplasm, since they are negative acute phase proteins $[1,9,12]$.

In this study, the histological atypicality of the splenic tumor was noteworthy, in which erythrophagocytosis and hemosiderin were not observed inside macrophages. The histologic characteristics described in HHS generally present marked erythrophagocytosis and histiocytes containing large deposits of hemosiderin $[4,9]$. The other histological changes observed in this case suggest the diagnosis of HS, but the confirmatory diagnosis can only be made through immunophenotypic characterization of the tumor $[3,8]$.

HHS, in this case, occurred in a 4-year-old Shih-Tzu. Although there are few reports of this neoplasm, in 2 studies, HHS occurred in elderly Rottweiler dogs $[5,12]$.

HHS is among the histiocytic disorders with the worst prognosis due to their aggressive biological behavior and non-specific clinical manifestations that hinder early diagnosis $[7,8]$. The patient in this report had a survival of 26 days from the first visit, which corroborates the reported survival rates ranging from days to 1 or 2 months, regardless of therapy $[3,4,6]$, which is usually palliative due to the rapid evolution of the tumor.

In conclusion, HHS is a rare and poorly described neoplasm in dogs with clinical signs and unspecific hematological alterations. Despite racial predisposition, dogs of any breed can develop tumors. HHS has an aggressive biological behavior, and treatment is only palliative.

\section{MANUFACTURERS \\ ${ }^{1}$ EasyPath|Diagnósticos. Indaiatuba, SP, Brazil. ${ }^{2}$ Dako North America Inc. Carpinteria, CA, USA.}

Acknowledgments. This study was financed in part by the Coordenação de Aperfeiçoamento de Pessoal de Nível Superior - Brasil (CAPES) - Finance Code 001. This research was also financially supported by Fundação de Amparo à Pesquisa e Inovação do Espírito Santo - FAPES.

Declaration of interest. The authors report no conflict of interest. The authors alone were responsible for the content and writing of paper.

\section{REFERENCES}

1 Abadie J., Hédan B., Cadieu E., De Brito C., Devauchelle P., Bourgain C., Parker H. G., Vaysse A., MargaritteJeannin P., Galibert F., Ostrander E. A. \& André C. 2009. Epidemiology, pathology, and genetics of histiocytic sarcoma in the Bernese mountain dog breed. The Journal of Heredity. 100(1): 19-27.

2 Affolter V.K. \& Moore P.F. 2002. Localized and Disseminated Histiocytic Sarcoma of Dendritic Cell Origin in Dogs. Veterinary Pathology. 39(1): 74-83.

3 Clifford C.A., Skorupski K.A. \& Moore P.F. 2020. Histiocytic Diseases. In: Vail D.M., Thamm D.H. \& Liptak J.M. (Eds). Small Animal Clinical Oncology. 6th edn. St. Louis: Elsevier, pp.791-810. 
4 Dervisis N.G., Kiupel M., Qin Q. \& Cesario L. 2017. Clinical prognostic factors in canine histiocytic sarcoma. Veterinary and Comparative Oncology. 15(4): 1171-1180.

5 Goulart J. C., Sanches F.J., Merlini N.B., Mazzucatto B.C., Gasser B. \& Marcusso P.F. 2020. Hemophagocytic Histiocytic Sarcoma in Dog. Acta Scientiae Veterinariae. 48(1): 574-580.

6 Jark P.C. \& Rodigheri S.M. 2016. Distúrbios Histiocíticos. In: Daleck C.R. \& De Nardi A.B. (Eds). Oncologia em Cães e Gatos. 2.ed. Rio de Janeiro: Editora Roca, pp.661-671.

7 Moore P.F. 2014. A Review of Histiocytic Diseases of Dogs and Cats. Veterinary Pathology. 51(1): 167-184.

8 Moore P.F. 2017. Canine and Feline Histiocytic Diseases. In: Meuten D.J. \& Wiley J. (Eds). Tumors in Domestic Animals. 5th edn. Ames: John Wiley \& Sons, pp.322-336.

9 Moore P.F., Affolter V.K. \& Vernau W. 2006. Canine hemophagocytic histiocytic sarcoma: A proliferative disorder of CD11d+ macrophages. Veterinary Pathology. 43(5): 632-645.

10 Mullin C. \& Clifford C.A. 2019. Histiocytic Sarcoma and Hemangiosarcoma Update. Veterinary Clinics of North America: Small Animal Practice. 49(5): 855-879.

11 Purzycka K., Peters L.M., Elliott J., Lamb C.R., Priestnall S.L., Hardas A., Johnston C.A. \& Rodriguez-Piza I. 2020. Histiocytic sarcoma in miniature schnauzers: 30 cases. Journal of Small Animal Practice. 61(6): 338-345.

12 Vieira T.C., Telles L.F., Nakagaki K.Y.R. \& Cassali G.D. 2021. Clinic-Pathological Aspects of Spleen Hemophagocytic Histiocytic Sarcoma in a Dog. Acta Scientiae Veterinariae. 49(Suppl 1): 630. 6p. 